\title{
Effects of oropharyngeal administration of colostrum on the incidence of necrotizing enterocolitis, late-onset sepsis, and death in preterm infants: a meta-analysis of RCTs
}

\author{
Jiaxin $\mathrm{Tao}^{1} \cdot$ Jing $\mathrm{Mao}^{1} \cdot$ Jixin $\mathrm{Yang}^{2} \cdot$ Yanwei Su$^{1}$ \\ Received: 13 September 2019 / Revised: 5 December 2019 / Accepted: 18 December 2019 / Published online: 3 January 2020 \\ (c) The Author(s), under exclusive licence to Springer Nature Limited 2020
}

\begin{abstract}
Necrotizing enterocolitis (NEC) and late-onset sepsis (LOS) are two major contributors to death among preterm infants. Oropharyngeal administration of colostrum (OAC) has been proved as an easy, safe, and economically viable technique to help preterm neonates to build up their immunity. In this review, we assessed the effects of OAC on preterm infants. Several mainstream databases were searched including PubMed, EMBASE, Cochrane Central Register of Controlled Trials (CENTRAL), and a website of clinical trials. Randomized controlled trials (RCTs) comparing OAC vs. placebo or no intervention in preterm infants (gestation age $<34$ weeks or birth weight $<1500 \mathrm{~g}$ ) were eligible. Overall, nine RCTs $(n=$ 689 ) were included in the review. Meta-analysis showed no statistical significance in terms of the incidence of NEC $(\mathrm{RR}=$ $0.59,95 \% \mathrm{CI}=0.33-1.06, p=0.08), \mathrm{LOS}(\mathrm{RR}=0.78,95 \% \mathrm{CI}=0.60-1.03, p=0.08)$ and mortality rate $(\mathrm{RR}=0.63,95 \%$ $\mathrm{CI}=0.38-1.05, p=0.07)$. No significant difference was found in the subgroup analysis, apart from the group of the undeveloped region in NEC and mortality. In addition, time was significantly reduced in terms of achieving full enteral feeding $(\mathrm{MD}=-3.60,95 \% \mathrm{CI}=-6.55-0.64, p=0.02)$ and hospital stay $(\mathrm{MD}=-10.38,95 \% \mathrm{CI}=-18.47-2.29, p=$ 0.01). The results show that OAC does not reduce the incidences of NEC, LOS, and death in preterm infants, but there is a trend toward a positive effect. It is therefore recommended as routine care for preterm infants in the NICU.
\end{abstract}

\section{Introduction}

With the astronomical development of modern medicine, there have been great advances in the development of neonatal intensive care to save the lives of preterm infants $(<37$ weeks gestation) with critical diseases. However necrotizing enterocolitis (NEC), a kind of intestinal excessive inflammation, still remains a major contributor to mortality rates as high as $20-30 \%$ and has been confirmed

Supplementary information The online version of this article (https:// doi.org/10.1038/s41430-019-0552-4) contains supplementary material, which is available to authorized users.

Yanwei $\mathrm{Su}$

yanweisu@hust.edu.cn

1 School of Nursing, Tongji Medical College, Huazhong University of Science and Technology, Wuhan 430030 Hubei, China

2 Department of Pediatric Surgery, Tongji Hospital, Tongji Medical College, Huazhong University of Science and Technology, Wuhan 430030, China as a multi-factor disease [1, 2]. Immature intestinal development, abnormal bacterial colonization, inappropriate feeding, and inflammatory cascades are all responsible for triggering of NEC [3, 4]. The disease has a blurry onset and a rapid progression making it undetectable in the early stages. Starting with feeding intolerance or abdominal distention, it will gradually evolve into catastrophic complications such as sepsis, disseminated intravascular coagulation, and death [4].

Late-onset sepsis (LOS), one of the major contributor to death of preterm infants in neonatal intensive care unit (NICU), is defined as infants with pathogen isolated from the blood culture drawn $\geq 72 \mathrm{~h}$ postnatally and pathogen based antibiotic treatment was continued for $\geq 5$ days [5], with morbidity and mortality rates of $20-38 \%$ and $13-19 \%$, respectively [5]. Several neonatal diseases have been identified as risk factors for the development of LOS, including NEC [6].

Colostrum, also called liquid gold, is produced in the first few days postpartum and is rich in immunoactive components dynamically switching in accordance with the mothers' condition; it is the best food for infants to build up 
their immunity [7]. Numerous studies have focused on the value of colostrum in terms of preventing, improving and curing diseases [8-11].

Anatomically, tonsils, adenoids, and lingual nodules form into the oropharyngeal-associated lymphoid tissue (OFALT), known as Waldeyer's ring, in the oropharyngeal cavity [12]. It becomes the first safeguard to regulate the entry of mother's milk, bacteria and, drugs that will directly interact with the immune cells of the OFALT [12]. At the same time, the OFALT also cooperates with gut lymphocytes by the activation of T-lymphocytes as messengers to attract more lymphocytes releasing anti-inflammatory cytokines [13, 14].

Oropharyngeal administration of colostrum (OAC) is a simple feeding method for preterm neonates first suggested by Rodriguez and in the following experiment, its safety and feasibility have been tested $[15,16]$. A small amount of colostrum, usually $0.2 \mathrm{ml}$, is perfused into a syringe (in vast majority studies) or soaked onto a cotton swab (in very few studies) and the infant's mouth is equally smeared with colostrum from the left buccal mucosal tissue to the right buccal mucosal tissue $[17,18]$. Colostrum is rich in various immunological substances, and therefore, OAC should be able to activate the oral and intestinal immunity, protecting the preterm infants against serious inflammatory diseases, such as NEC and LOS [19-21].

To confirm OAC's benefits on preterm babies, a large number of studies (RCTs or non-RCTs) have been conducted. Low-birth-weight infants $(<1500 \mathrm{~g})$ or inadequate gestation age (GA) babies $(<34$ weeks) were chosen, with $\mathrm{OAC}$ as an intervention with placebo or without intervention, and the biochemical outcomes (bacterial colonization), the clinical outcomes (LOS, NEC, death, etc.) and the nutrition outcomes (weight at discharge, day feedings initiation, etc.) were measured [22]. Abd-Elgawad et al. found that OAC could shorten the duration of hospital stays and meet the early achievement of feeding, albeit without any decline in the incidence of NEC [22]. However, Zhang et al. have discovered that OAC has potential protection on NEC with statistical significance showing [23]. Some nonRCT studies have also provided some information about the benefits of OAC [24-26].

Previous systemic reviews and meta-analyses have shown that OAC did not have any influence on the clinical outcomes (NEC, LOS, and mortality). However these studies were limited by RCTs of small sample size and the inclusion of non-RCT studies $[27,28]$. In this context, the objective of the present review was to assess the effects of OAC on preterm infants by updating the systemic review on this topic. Nine RCTs, including 689 participants, were enrolled; in addition, another two important health outcomes, including time to reach full enteral feeding and duration of hospital stay, were assessed. The outcomes of this meta-analysis provide important information for the further development of clinical practice.

\section{Method}

This meta-analysis was drafted in the requirements of the Preferred Reporting Items for Systemic Reviews and MetaAnalyses [29]. Since all data used in this review were acquired from previously published articles, no ethical approval or patient consent was required.

\section{Search strategies}

We systematically searched the original articles published in PubMed, EMBASE, Cochrane Central Register of Controlled Trials (CENTRAL), and website of the clinical trials from the establishment of the databases or websites up to May 31, 2019. The search strategy used a different combination of MeSH terms and keywords following the PICO principle. The search terms used were ("preterm infant" OR "premature infant" OR "neonatal prematurity") AND ("colostrum" OR "oropharyngeal administration") AND ("NEC" OR "sepsis"). In addition, the references of articles were also screened for a comprehensive assessment.

\section{Inclusion and exclusion criteria}

The eligible studies had to meet the following criteria: (1) study type is RCT; (2) participants are preterm infants whose GA was $<34$ weeks or with a birth weight (BW) $<1500 \mathrm{~g}$; (3) the RCTs focused on the comparison of OAC as monotherapy with saline or normal sterile water or no intervention for the observation of the NEC rate; (4) primary outcome including NEC stage $\geq 2$ (Bell staging criteria), LOS are reported; (5) articles are written in English. The exclusion criteria were as follows: (1) non-human studies; (2) reviews, abstracts, letters, commentaries, editorials, and case reports; (3) grey literature and conference abstract are not considered.

\section{Study selection}

First, all records were imported into a document management software, and duplicated literatures were electronically removed. The title and abstract of the initial broad search results were screened to identify the potentially eligible studies by the two independent reviewers Yanwei $\mathrm{Su}$ and Jiaxin Tao. After that, full-text articles were downloaded, read, and assessed using the confirmed criteria for exclusion or inclusion. In the case of different opinions, discrepancies were solved by discussions or by inviting a third reviewer. 


\section{Data extraction}

Data were extracted by using a predefined data collection form, which contained the following information: basic characteristics (author, year, country, and sample size), patients' characteristics (GA and $\mathrm{BW}$ ), intervention protocols (dosage, interval, duration, and control), primary outcomes (incidence of NEC, LOS, and mortality rate) and secondary outcomes (time to reach full enteral feeding and duration of hospital stay). Data extraction was conducted by Yanwei Su and Jiaxin Tao, and the third reviewer Jing Mao scrutinized and validated the final data form.

\section{Risk assessment of Bias of RCT}

To determine validity, the quality of each study was evaluated by using the Cochrane risk of the Bias Assessment Tool to assess Risk of Bias (ROB) by the two reviewers Yanwei Su and Jiaxin Tao [30]. The Cochrane risk of the Bias Assessment Tool contained six domains, namely random sequence generation, allocation concealment, blinding of intervention and outcome assessors, completeness of follow up, selectivity of reporting and other potential sources of bias, which were all categorized as low, high, or unclear according to the Cochrane Collaboration guidelines [30].

\section{Statistical analysis}

Review Manager (version 5.3; Cochrane Collaboration, Nordic Cochrane) was used to perform statistical analysis. Relative ratio (RR) was calculated for dichotomous outcome, while mean difference (MD) was used for the continuous outcome. For both outcomes, 95\% confidence intervals (CI) were considered. Statistical heterogeneity was estimated using the Chi-square based $\mathrm{Q}$ test with $p$ value and $I^{2}$ statistic displaying. The value of $I^{2}$ was categorized into low $(0-40 \%)$, moderate (30-60\%), substantial $(50-90 \%)$, and considerable $(75-100 \%)$ to evaluate the heterogeneity among studies [31]. If $I^{2}<50 \%$, the fix-effect model was used, if not, the random-effect model was chosen, considering the significant heterogeneity among the studies. Subgroup analysis was required to find the source of heterogeneity by classifying different studies. Sensitivity analysis was also conducted to testify the stability of the pooled results. Publication bias was assessed by making a funnel plot using Egger's test if at least ten studies were included in this meta-analysis [32].

\section{Quality of evidence assessment}

According to the Grades of Recommendation, Assessment, Development, and Evaluation (GRADE) guidelines, the quality of each pooled results was rated as very low, low, moderate and high. The RCTs were first considered as high quality, but could be downgraded by the following factors: (1) ROB, (2) inconsistency, (3) indirectness, (4) imprecision, and (5) publication bias [31].

\section{Results}

\section{Study selection and characteristics}

Initially, 114 records were retrieved from all databases and imported into a document management software; duplicated studies were mechanically deleted, leaving 56 studies. Of these, 38 were excluded through title or abstract reading and another two studies were identified through citation tracking after full-text reading. Eleven studies were excluded for the following reasons: (1) proceedings, no full-text $(n=4)$; (2) non-RCTs $(n=5)$; (3) other reasons $(n=2)$. Finally, nine RCTs met the inclusion criteria of this review. A study selection flow chart (Fig. 1) was made and displayed the whole process.

In total, 689 preterm infants were enrolled in the review, while oropharyngeal administration of mother's colostrum worked as an intervention to assess its effect on clinical or biochemical outcomes. GA and BW were $<34$ weeks and $1500 \mathrm{~g}$, respectively, meeting the standards of preterm neonates. Infants with congenital anomalies, chromosomal abnormalities, hepatitis B virus (HBV), or human immunodeficiency virus (HIV) infections were excluded from the study. In all studies, $0.2 \mathrm{ml}$ of mother's colostrum was applied as a single administration amount, with a feeding time ranging from $24 \mathrm{~h}$ after postnatal life to days up to 7

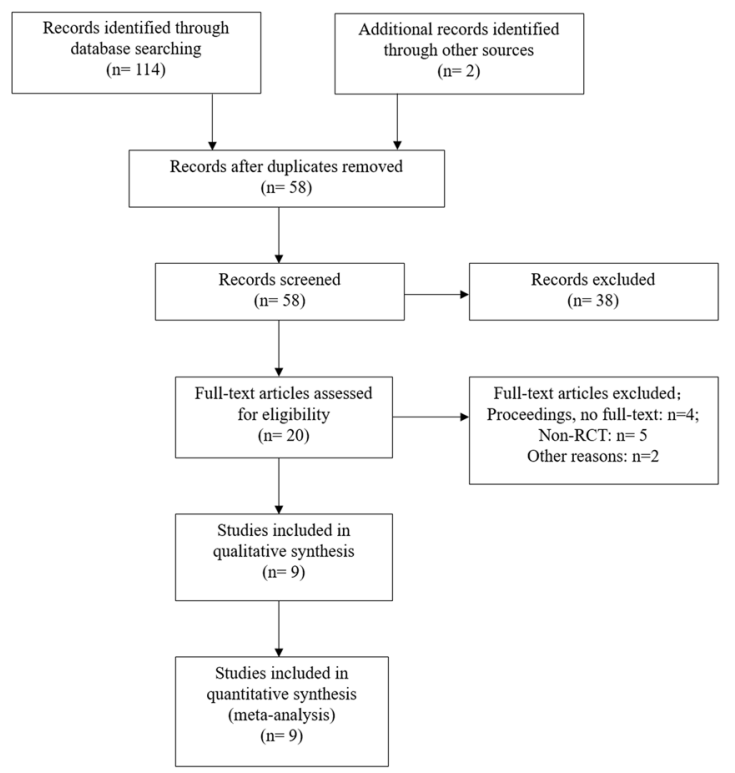

Fig. 1 Flow diagram of search strategy and study selection. 


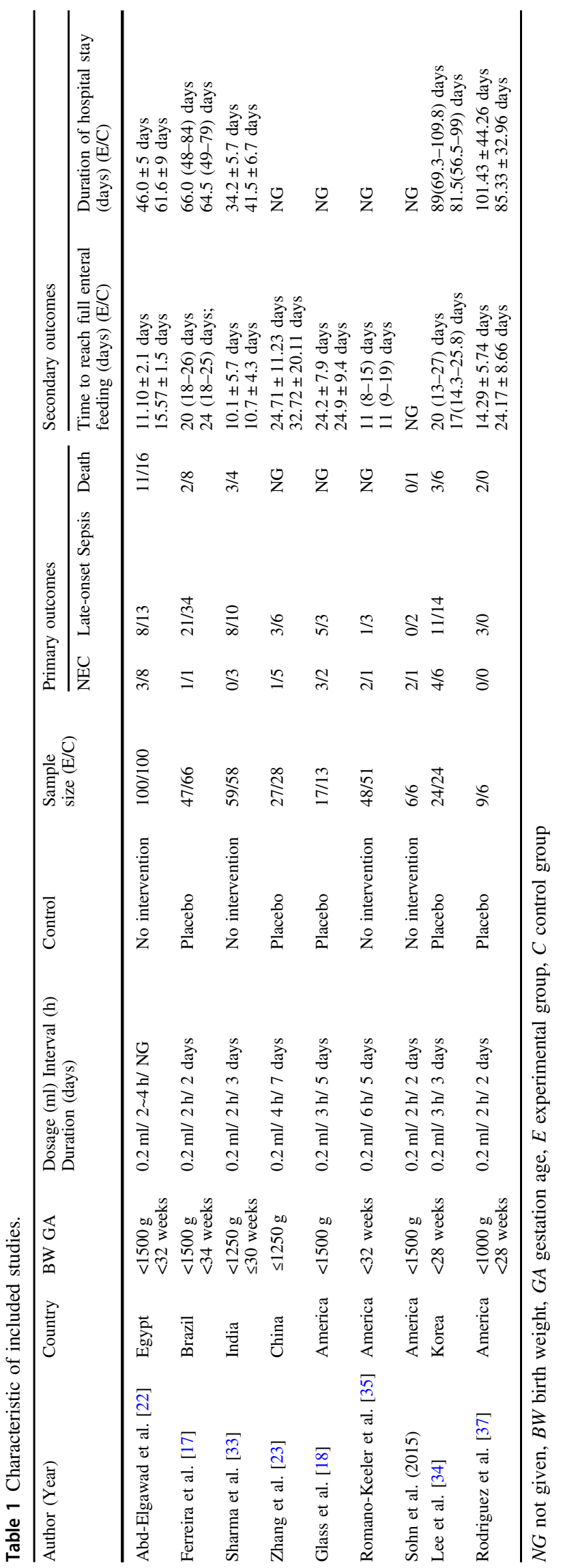

and a giving interval set to 2 to $6 \mathrm{~h}$. Normal saline, sterile water, or regular feeding were used as a control. The primary outcomes were the incidence of NEC (Bell's stage $\geq 2$ ), the rate of LOS, and all-cause mortality rate. Secondary outcomes consisted of the time to reach to full eternal feeding and the duration of the hospital stay. The details of the included studies are summarized in Table 1.

\section{ROB of RCT}

A summary of ROB of RCT (Table 2) is provided below. Methodological weaknesses existed for most of the included studies. Six studies [17, 18, 22, 23, 33, 34] had a low ROB in random sequence generation, with one study [35] reported a high risk and two [36, 37] reported an unclear risk. Allocation concealment was of high risk in one study [35] and of unclear risk in three studies $[17,18,37]$. The risk of blinding of participants and personnel was high in four studies [18, 33, 35, 36] and unclear in one study [37]. Similarly, four studies $[18,33,35,36]$ were also of high ROB in blinding of the outcome assessment, with two studies [23, 37] of unclear risk. Low ROB was mainly in incomplete outcome data and selective reporting, while unclear risk appeared in other bias. To better identify the ROB of the included studies, we generate a graph presenting the percentages for each domain and the outcome of each ROB assessment are displayed in Fig. 2.

\section{Effect on Incidence of NEC}

Nine studies, with a total sample of 689 preterm infants, measured the incidence of NEC (Fig. 3a). The NEC rate was $4.7 \%$ in the OAC group compared with $7.7 \%$ in the control group. The pooled result showed no statistical significance between the OAC group and the control group ( $p=0.08, \mathrm{RR}=0.59,95 \% \mathrm{CI}=0.33-1.06$ ). The risk of developing $\mathrm{NEC}$ in the OAC group was $41 \%$ lower than in the control group. The fixed-effect model was used due to a low heterogeneity among studies $\left(I^{2}=\right.$ $0 \%, p=0.56)$. Subgroup analysis was conducted and no statistical significance was found apart from the subgroup of the undeveloped region $\left(I^{2}=0 \%, p=0.02\right)$.

\section{Effect on the Rate of LOS}

Nine studies, with a total sample of 689 preterm infants were deemed available for meta-analysis to measure the rate of LOS (Fig. 3b). Sepsis rate was $17.8 \%$ in the OAC group compared with $24.1 \%$ in the control group. There was no statistical significance between the OAC group and the control group $(p=0.08, \mathrm{RR}=0.78,95 \% \mathrm{CI}=$ $0.60-1.03)$. The risk of developing LOS in the OAC 
Table 2 Risk of bias of included studies.

\begin{tabular}{|c|c|c|c|c|c|c|c|}
\hline Studies & $\begin{array}{l}\text { Random } \\
\text { sequence } \\
\text { generation } \\
\text { (selection bias) }\end{array}$ & $\begin{array}{l}\text { Allocation } \\
\text { concealment } \\
\text { (selection bias) }\end{array}$ & $\begin{array}{l}\text { Blinding of } \\
\text { participants and } \\
\text { personnel } \\
\text { (performance bias) }\end{array}$ & $\begin{array}{l}\text { Blinding of } \\
\text { outcome } \\
\text { assessment } \\
\text { (detection bias) }\end{array}$ & $\begin{array}{l}\text { Incomplete } \\
\text { outcome data } \\
\text { (attrition bias) }\end{array}$ & $\begin{array}{l}\text { Selective } \\
\text { reporting } \\
\text { (reporting bias) }\end{array}$ & Other bias \\
\hline $\begin{array}{l}\text { Abd- } \\
\text { Elgawad et al }\end{array}$ & Low risk & Low risk & Low risk & Low risk & Low risk & Low risk & Low risk \\
\hline Ferreira et al. & Low risk & Unclear risk & Low risk & Low risk & Low risk & Low risk & Low risk \\
\hline Sharma et al. & Low risk & Low risk & High risk & High risk & Low risk & Low risk & Unclear risk \\
\hline Zhang et al. & Low risk & Low risk & Low risk & Unclear risk & Low risk & Low risk & Unclear risk \\
\hline Glass et al. & Low risk & Unclear risk & High risk & High risk & Low risk & Low risk & Unclear risk \\
\hline $\begin{array}{l}\text { Romano- } \\
\text { Keeler et al. }\end{array}$ & High risk & High risk & High risk & High risk & Low risk & Low risk & Unclear risk \\
\hline Sohn et al. & Unclear risk & Low risk & High risk & High risk & Low risk & Low risk & Unclear risk \\
\hline Lee et al. & Low risk & Low risk & Low risk & Low risk & Low risk & Low risk & Low risk \\
\hline Rodriguez et al. & Unclear risk & Unclear risk & Unclear risk & Unclear risk & Low risk & Low risk & Unclear risk \\
\hline
\end{tabular}

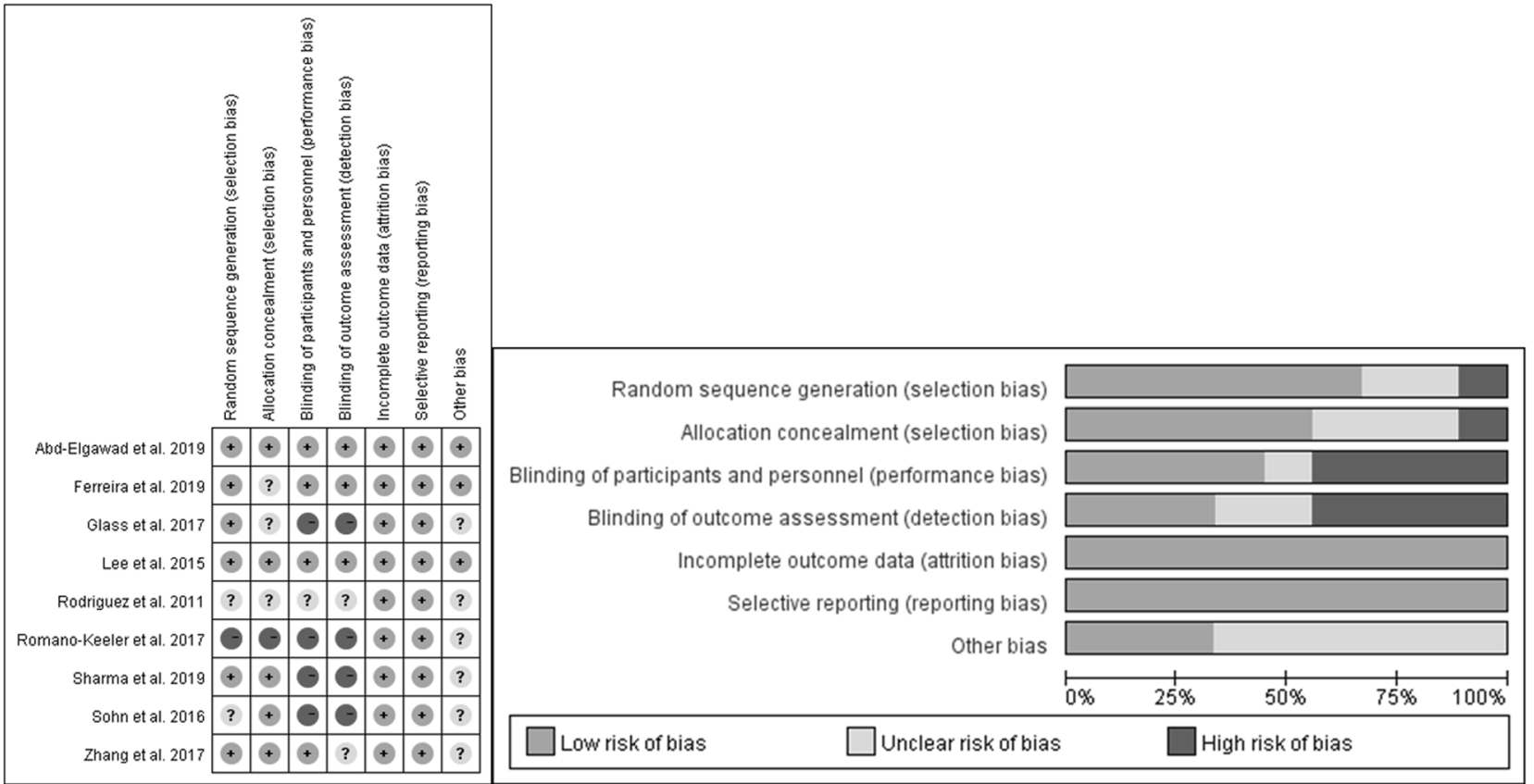

Fig. 2 Summary of risk of bias.

group was $22 \%$ lower than in the control group. The fixedeffect model was used due to low heterogeneity among studies $\left(I^{2}=0 \%, p=0.80\right)$. No statistical significance was found in the subgroup analysis.

\section{Effect on the all-cause mortality rate}

After eliminating the studies of Zhang et al. [23], Glass et al. [18], and Romano-Keeler et al. [35] for the all-cause mortality rate, six studies [17, 22, 33, 34, 36, 37], with a total sample of 505 preterm infants were included to measure the mortality rate (Fig. 3c). The rate was $8.6 \%$ in the OAC group compared with $13.5 \%$ in the control group.
There was no statistical significance showing that OAC could reduce the mortality rate $(p=0.07, \mathrm{RR}=0.63,95 \%$ $\mathrm{CI}=0.38-1.05)$. The risk of death in the OAC group was $37 \%$ lower than in the control group. The fixed-effect model was used due to the low heterogeneity among studies $\left(I^{2}=\right.$ $0 \%, p=0.80$ ). No statistical significance was found in the subgroup analysis apart from the group of the undeveloped region.

\section{Time to reach full enteral feeding}

Five studies [18, 22, 23, 33, 37], with a sample of 417 preterm infants, were included (Fig. 4a). In another 


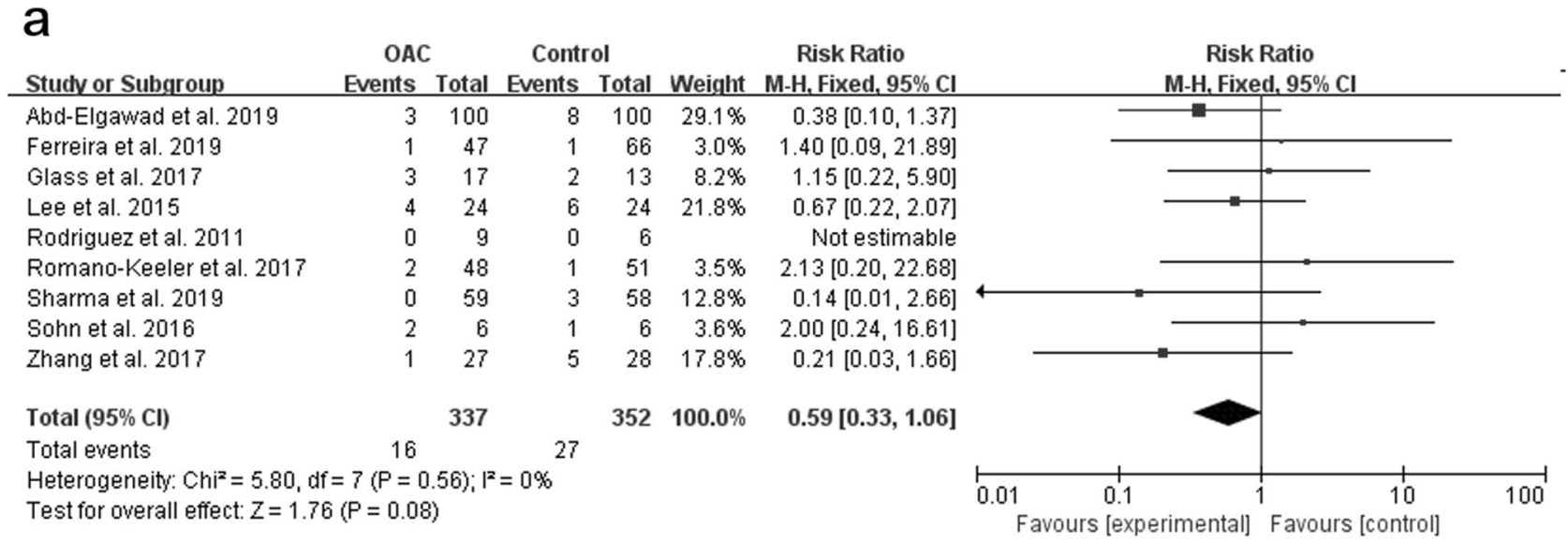

b

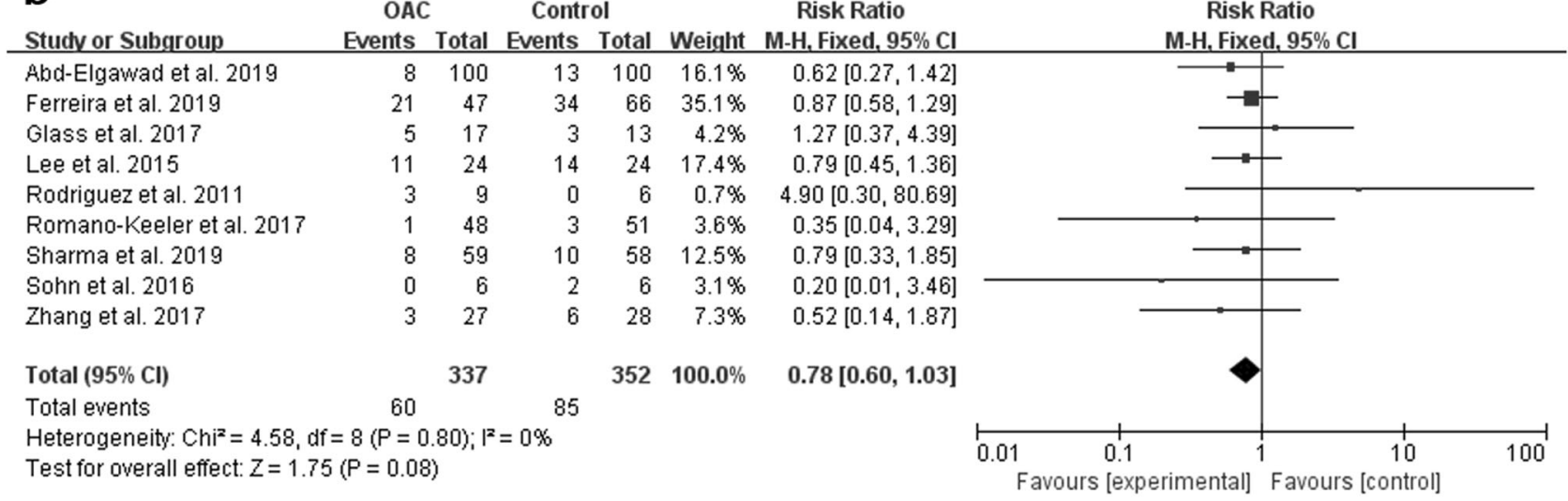

\section{C} OAC Control Risk Ratio Risk Ratio

Study or Subgroup Abd-Elgawad et al. 2019 Ferreira et al. 2019 Lee et al. 2015 Rodriguez et al. 2011 Sharma et al. 2019 Sohn et al. 2016

Events

Total $(95 \% \mathrm{Cl})$

Total events

Heterogeneity: $\mathrm{Chi}^{2}=2.34, \mathrm{df}=5(\mathrm{P}=0.80) ; \mathrm{I}^{2}=0 \%$

Test for overall effect: $Z=1.79(P=0.07)$

Conts Total Weight M-H, Fixed, 95\% Cl M-H, Fixed, 95\% Cl

$0.69[0.34,1.41]$

$0.35[0.08,1.58]$

$0.50[0.14,1.77]$

$3.50[0.20,62.27]$

$0.74[0.17,3.15]$

$0.33[0.02,6.86]$

$260 \quad 100.0 \% \quad 0.63[0.38,1.05]$

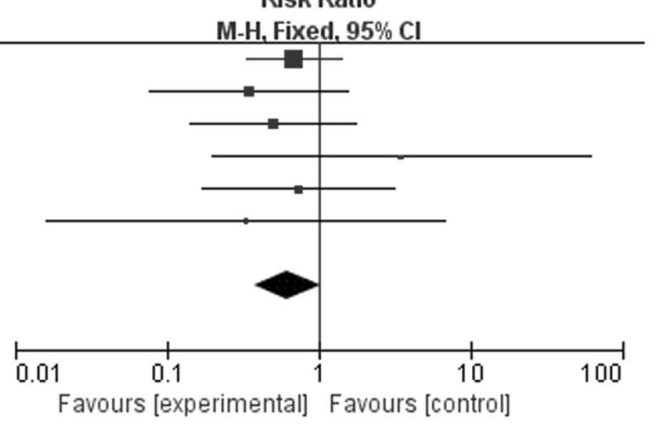

Fig. 3 Forest plot of primary outcomes. a Effect of OAC on NEC. b Effect of OAC on LOS. c Effect of OAC on death.

three $[17,34,35]$ studies, the item "time to reach to full enteral feeding" was reported in the form of median and interquartile range, considering the small sample size, data transformation was not performed, and one [36] study did not report this outcome. The pooled results showed statistical significance between the OAC group and the control group $(p=0.02, \mathrm{MD}=-3.60$, 95\% CI $=-6.55-0.64)$. The random-effect model was used to estimate the combined result due to the strong heterogeneity among studies $\left(I^{2}=80 \%, p<\right.$ 0.001 ).

\section{Duration of hospital stay}

Duration of hospital stay was reported in five studies $[17,22,33,34,37]$, while two studies [17, 34] were excluded; thus, three studies [22, 33, 37], with a sample of 332 preterm infants, were included (Fig. 4b). The pooled results showed statistical significance between the OAC group and the control group $(p=0.01, \mathrm{MD}=-10.38,95 \%$ $\mathrm{CI}=-18.47-2.29)$. The random-effect model was used to estimate the combined result due to the strong heterogeneity among studies $\left(I^{2}=94 \%, p<0.001\right)$. 

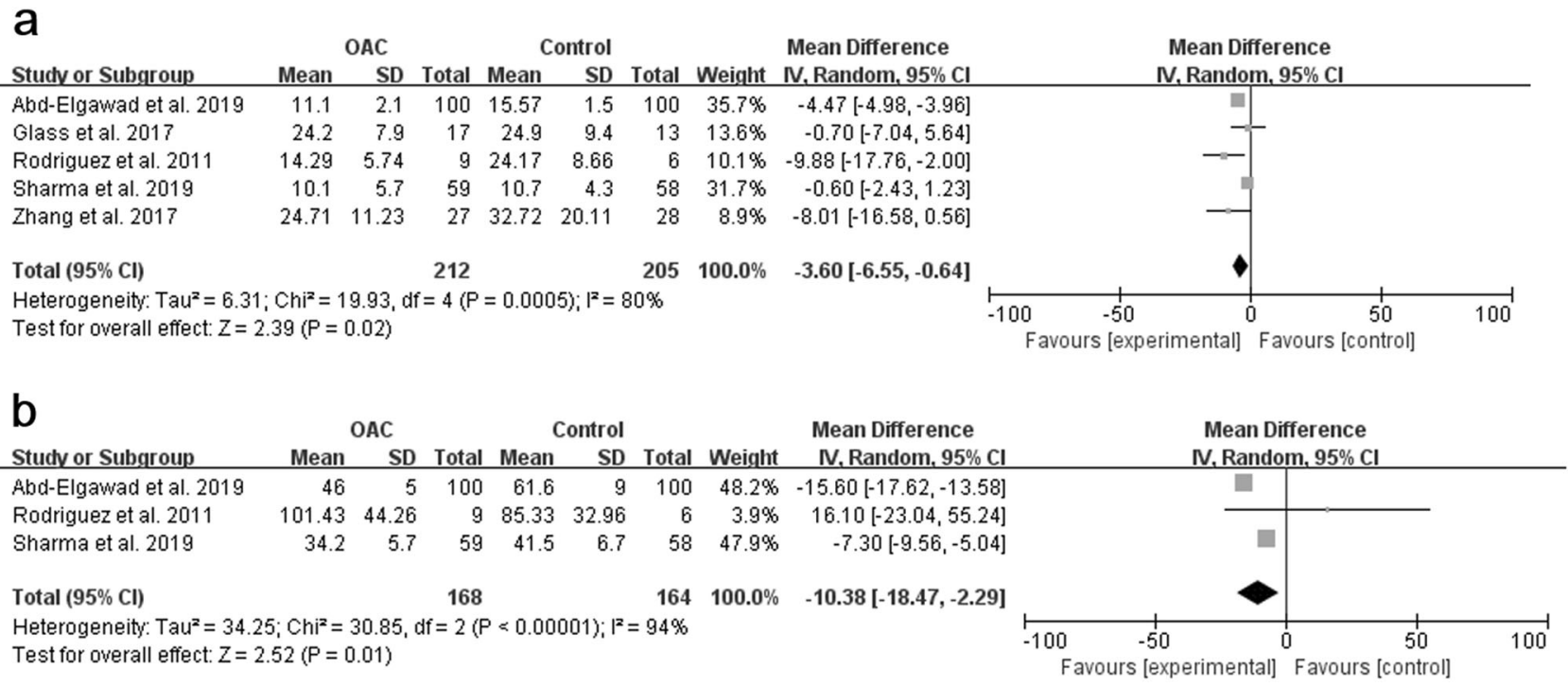

Fig. 4 Forest plot of secondary outcomes. a Effect of OAC on time to reach full enteral feeding. b Effect of OAC on duration of hospital stay.

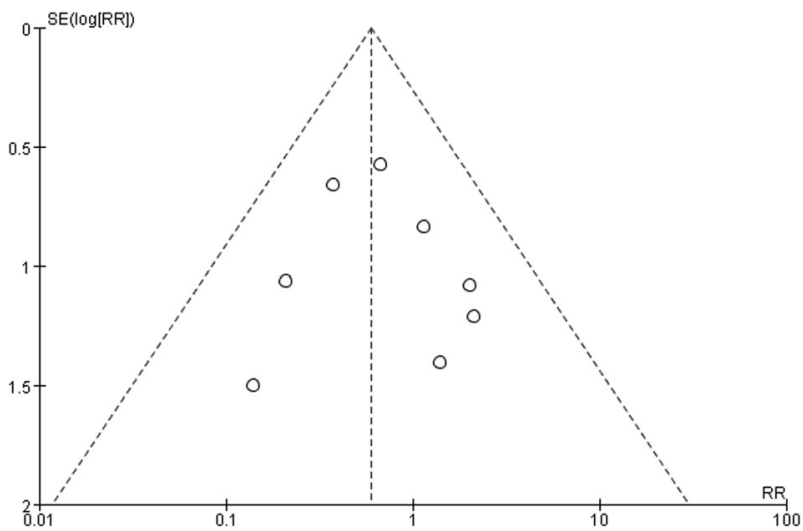

Fig. 5 Funnel plot of publication bias.

\section{Publication bias}

Publication bias could not be assessed properly by visual inspection due to insufficient studies. (Fig. 5).

\section{Quality assessment}

The overall quality of evidence across all the RCTs for NEC, LOS, and mortality rate was moderate by using the GRADE approach (Table 3). The rating was downgraded by imprecision of outcomes due to the wide $95 \%$ CI.

\section{Discussion}

Although modern medicine has made great advances, NEC and LOS are still devastating inflammatory diseases among preterm infants, with significant mortality rates. The mechanism of NEC is still unclear, and multiple preventive strategies have been developed and tested to examine the effects of probiotic or amino acid supplementation and antibiotic treatment. Recently, some studies have focused on the efficacy of colostrum, which is rich in bioactive factors. A retrospective cohort study has shown the significantly lower rates of NEC and LOS and earlier successful enteral feeding in preterm infants who received a standardized feeding protocol which included OAC [38]. However, some other articles have concluded that OAC had no influence on the common neonatal morbidities [25, 26]. Despite of the contradictory conclusions, OAC still has beneficial effects on preterm infants.

The key results of previous studies in this field need to be discussed. Some studies found that colostrum was effective in reducing the rate of NEC, revealing its effect in building up immunity [11, 39-42]. Term babies could suck their mothers' nipples to obtain breast milk and their immunity would be triggered through the first bite with colostrum being served as stimulator [43, 44]. However, preterm infants who have undeveloped digestive systems are deprived of this natural process because they have to be breastfed via a nasogastric tube that bypasses their oropharynx. Some researchers found that the oral cavities of ill babies are easily colonized by pathogens after being administrated into the NICU and the use of oral rinses such as chlorhexidine, could bring unpleasant adverse effects $[45,46]$. Thus, oral priming with colostrum is a better way to help preterm infants to strengthen their immunity, thereby preventing infections.

The pooled results of our current review are in contrast to what we have predicted in theory. The subgroup analysis did not deliver any positive results apart from the group of 
Table 3 Quality assessment according to GRADE guidelines.

\begin{tabular}{|c|c|c|c|c|c|c|c|c|}
\hline \multirow[t]{2}{*}{ Outcome } & \multirow{2}{*}{$\begin{array}{l}\text { Sample size/no. } \\
\text { of studies }\end{array}$} & \multicolumn{5}{|c|}{ Quality assessment } & \multirow{2}{*}{$\begin{array}{l}\text { Relative effect, RR } \\
(95 \% \text { CI })\end{array}$} & \multirow[t]{2}{*}{ Quality } \\
\hline & & $\begin{array}{l}\text { Risk of } \\
\text { bias }\end{array}$ & Inconsistency & Indirectness & Imprecision & Publication bias & & \\
\hline NEC & $\begin{array}{l}689 \\
9\end{array}$ & $\begin{array}{l}\text { Not } \\
\text { serious }\end{array}$ & Not serious & Not serious & Serious $^{\mathrm{a}}$ & Undetected & $0.59(0.33-1.06)$ & Moderate \\
\hline $\begin{array}{l}\text { Late- } \\
\text { onset Sepsis }\end{array}$ & $\begin{array}{l}689 \\
9\end{array}$ & $\begin{array}{l}\text { Not } \\
\text { serious }\end{array}$ & Not serious & Not serious & Serious $^{\mathrm{a}}$ & Undetected & $0.78(0.60-1.03)$ & Moderate \\
\hline Mortality & $\begin{array}{l}505 \\
6\end{array}$ & $\begin{array}{l}\text { Not } \\
\text { serious }\end{array}$ & Not serious & Not serious & Serious $^{\mathrm{a}}$ & Undetected & $0.63(0.38-1.05)$ & Moderate \\
\hline
\end{tabular}

${ }^{\mathrm{a}} 95 \% \mathrm{CI}$ is wide enough which contains $\mathrm{RR}=1$

undeveloped (Supplemental Table 1). The potential reasons of these negative results were explored to address this issue.

First of all, OAC does not have a unified terminology, which means that no standard operation is set, and the initial time of OAC varies in interval and duration. Immunity building is a long-term process, and OAC should be continued over longer periods to determine its effects. For example, Glass et al., using OAC intervention for 5 days, found that the level of sIgA of saliva increased statistically on day 7 but without a subsequent increase on day 14 [18]. This indicates that extended OAC treatment might provide sustained immune protection.

Second, colostrum was collected after the infants had been administered into the NICU. It was packed in a plastic bag, stored in a refrigerator and thawed at room temperature $[26,34,36]$. This approach might lead to the inactivation of some immune protective factors, resulting in decreased levels of active substances [44]. Contamination might also occur, as some studies have shown pathogenic organisms in mother milk samples [47-49].

Third, NEC and LOS are not single-factor diseases and might be triggered and influenced by multiple factors such as the status of preterm infants, the therapy received and the medical condition, which are all significant [50].

Some studies have a moderate to strong bias, which might influence the outcome. All included RCTs are singlecenter studies, and most of them have a relatively small sample size and, therefore, limited power. For these reasons, a 5 years, multicenter, double-blind RCT has been designed to evaluate the safety and efficacy in reducing the incidence of NEC, LOS, and death in preterm infants [51]. This trial is still ongoing, and the results will bring robust evidence to address this issue.

Although our review focusing on the effects of OAC on the incidence of NEC, LOS, and death showed negative outcomes, there are still some evidences concerning the effects of OAC as a beneficial trial for preterm infants. Achieving full enteral feeding is an important health outcome since it is related to the removal of the central line, possibly, to lower infection rates [52]. The duration of hospital stay reflects the condition of preterm infants and the related medical costs, and the pooled results indicate that OAC would shorten the time to achieve these two goals. Some other clinical or biochemical outcomes in these RCT studies still need to be discussed. Abd-Elgawad et al. have reported lower growth rates of Klebsiella species in the oropharyngeal pouch, a lower incidence of ventilatorassociated pneumonia, a shorter duration of oxygen therapy and, fewer episodes of feeding intolerance after OAC treatment [22]. In another study, Zhang et al. have reported that OAC could raise the level of lactoferrin in saliva after 7 days and 21 days, while Glass et al. have also found increased sIgA levels of saliva at day 7 [18, 23]. Sohn et al. have reported changes in the oral microbiota colonization in the OAC group [36], while Lee et al. report a decreased level of pro-inflammatory cytokines and an increased level of immune-protective factors compared with the control group [34]. Overall, these results suggest the potential positive effects of OAC on the biochemical outcomes, without any adverse effects.

The strength of this review includes several aspects. We used a systemic, comprehensive and broad searching strategy with hand searching of some references. Robust methodologies were adopted including explicit inclusion and exclusion criteria, independent data extraction, ROB assessment, quality assessment, and subgroup analysis. The latest published RCT studies with moderate sample size and the value of $I^{2}$ being zero increase the validity of the results in comparison to previous reviews.

However, our review has also considerable limitations. First, there is an inconsistency in the given interval and duration of the OAC treatment in the included studies. Second, the total number of participants was moderate, whereas the incidence of NEC and LOS was low. The ongoing trial with a sample size of 622 will, however, support our results [51]. Third, the incidences of NEC, LOS, and death were not the primary outcomes in some studies. Some data related to the secondary outcomes were inadequate, with strong heterogeneity. Thus, the pooled results of the secondary outcomes should be interpreted 
with caution. In view of these limitations, larger, well-designed RCTs are needed, since some included RCT studies had a high ROB and did not contain their predesigned study protocols.

Up to now, preterm birth is still the major cause of multiple life-threatening diseases, and the economic costs are high. Although a significant improvement in the clinically important outcomes was not observed in the OAC group, OAC can still have positive effects on preterm infants. It is an easy, safe, and economical technique and has been confirmed by a large number of studies, lending itself as routine care for preterm infants administered into NICU.

For clinical staff, we advise to improve the OAC protocol as follows: (1) OAC could start earlier, at a higher frequency and duration to further boost infant immunity; (2) aseptic principles must be kept in mind in every nursing step to prevent infections; (3) fresh colostrum is better than long-term stored one, mainly because some bioactive factors might get inactivated in the process.

For researchers, the pooled results also provide implications for future studies, with the following recommendations: (1) the registration of clinical trials with detailed protocols on the website and reported in articles; (2) the standardization of OAC protocols to better understand the effects of OAC; (3) the development of high-quality RCTs with large sample sizes and low ROB; (4) the effect of OAC is not limited to NEC and LOS, and future studies might focus on its effect on other neonatal diseases.

\section{Conclusions}

There is evidence that OAC could not significantly reduce the incidences of NEC, LOS, and death, but there is a trend of positive effects. The OAC might reduce the time to achieve full enteral feeding and reduce hospital stay. Future studies with large sample sizes and a high quality are needed to confirm the effects of OAC. For now, OAC is still recommendable as a routine nursing care for preterm infants administered into the NICU.

Acknowledgements YS contributed to the design of review protocol, define the research theme, screen the eligible studies, conduct data extraction, and revise the article. JT contributed to screen the eligible studies, conduct data extraction, analyze data, make the tables, and write the article. JM and JY contributed to interpret the results and give expert advice on the article.

Funding This research was funded by the National Natural Science Foundation of China (81601330) and Natural Science Foundation of Hubei Province (2019CFB516).

\section{Compliance with ethical standards}

Conflict of interest The authors declare that they have no conflict of interest.
Publisher's note Springer Nature remains neutral with regard to jurisdictional claims in published maps and institutional affiliations.

\section{References}

1. Lau PE, Cruz SM, Ocampo EC, Nuthakki S, Style CC, Lee TC, et al. Necrotizing enterocolitis in patients with congenital heart disease: a single center experience. J Pediatr Surg. 2018;53:914-7.

2. Neu J, Walker WA. Necrotizing enterocolitis. N Engl J Med. 2011;364:255-64.

3. Lim JC, Golden JM, Ford HR. Pathogenesis of neonatal necrotizing enterocolitis. Pediatr Surg Int. 2015;31:509-18.

4. Lin PW, Stoll BJ. Necrotising enterocolitis. Lancet. 2006;368: 1271-83.

5. El Manouni El Hassani S, Berkhout DJC, Niemarkt HJ, Mann S, de Boode WP, Cossey V, et al. Risk factors for late-onset sepsis in preterm infants: a multicenter case-control study. Neonatology. 2019;116:42-51.

6. Tsai MH, Hsu JF, Chu SM, Lien R, Huang HR, Chiang MC, et al. Incidence, clinical characteristics and risk factors for adverse outcome in neonates with late-onset sepsis. Pediatr Infect Dis J. 2014;33:e7-e13.

7. Cruz D, Bazacliu C. Enteral feeding composition and necrotizing enterocolitis. Semin Fetal Neonatal Med. 2018;23:406-10.

8. Cortez J, Makker K, Kraemer DF, Neu J, Sharma R, Hudak ML. Maternal milk feedings reduce sepsis, necrotizing enterocolitis and improve outcomes of premature infants. J Perinatol. 2018;38: $71-4$.

9. Maffei D, Schanler RJ. Human milk is the feeding strategy to prevent necrotizing enterocolitis! Semin Perinatol. 2017;41:36-40.

10. Meier PP. Human milk and clinical outcomes in preterm infants. Nestle Nutr Inst Workshop Ser. 2019;90:163-74.

11. Sun H, Han S, Cheng R, Hei M, Kakulas F, Lee SK. Testing the feasibility and safety of feeding preterm infants fresh mother's own milk in the NICU: a pilot study. Sci Rep. 2019;9:941.

12. Bocci V. Absorption of cytokines via oropharyngeal-associated lymphoid tissues. Clin Pharmacokinetics. 1991;21:411-7.

13. Brod SA. Gut response: therapy with ingested immunomodulatory proteins. JAMA Neurol. 1997;54:1300-2.

14. Butcher EC, Picker LJ. Lymphocyte homing and homeostasis. Science. 1996;272:60-7.

15. Rodriguez NA, Meier PP, Groer MW, Zeller JM. Oropharyngeal administration of colostrum to extremely low birth weight infants: theoretical perspectives. J Perinatol. 2009;29:1-7.

16. Rodriguez NA, Meier PP, Groer MW, Zeller JM, Engstrom JL, Fogg L. A pilot study to determine the safety and feasibility of oropharyngeal administration of own mother's colostrum to extremely low-birth-weight infants. Adv Neonatal Care. 2010;10: 206-12.

17. Ferreira D, Oliveira AMM, de Leves DV, de Bem EB, Fatureto GG, Navarro NF, et al. Randomized controlled trial of oropharyngeal colostrum administration in very-low-birth-weight preterm infants. J Pediatr Gastroenterol Nutr. 2019;69:126-30.

18. Glass KM, Greecher CP, Doheny KK. Oropharyngeal administration of colostrum increases salivary secretory IgA levels in very low-birth-weight infants. Am J Perinatol. 2017;34:1389-95.

19. Eid P, Meritet JF, Maury C, Lasfar A, Weill D, Tovey MG. Oromucosal interferon therapy: Pharmacokinetics and pharmacodynamics. J Interferon Cytokine Res. 1999;19:157-69.

20. Fleischmann WR Jr, Koren S, Fleischmann CM. Orally administered interferons exert their white blood cell suppressive effects via a novel mechanism. Proc Soc Exp Biol Med. 1992;201:200-7.

21. Tovey MG, Meritet JF, Guymarho J, Maury C. Mucosal cytokine therapy: Marked antiviral and antitumor activity. J Interferon Cytokine Res. 1999;19:911-21. 
22. Abd-Elgawad M, Eldegla H, Khashaba M, Nasef N. Oropharyngeal administration of mother's milk prior to gavage feeding in preterm infants: a pilot randomized control trial. JPEN J Parenter Enteral Nutr. 2019. https://doi.org/10.1002/jpen.1601. [Published online ahead of print 6 May 2019].

23. Zhang Y, Ji F, Hu X, Cao Y, Latour JM. Oropharyngeal colostrum administration in very low birth weight infants: a randomized controlled trial. Pediatr Crit Care Med. 2017;18:869-75.

24. Moreno-Fernandez J, Sanchez-Martinez B, Serrano-Lopez L, Martin-Alvarez E, Diaz-Castro J, Pena-Caballero M, et al. Enhancement of immune response mediated by oropharyngeal colostrum administration in preterm neonates. Pediatr Allergy Immunol. 2019;30:234-41.

25. Seigel JK, Smith PB, Ashley PL, Cotten CM, Herbert CC, King BA, et al. Early administration of oropharyngeal colostrum to extremely low birth weight infants. Breastfeed Med. 2013;8:491-5.

26. Snyder R, Herdt A, Mejias-Cepeda N, Ladino J, Crowley K, Levy P. Early provision of oropharyngeal colostrum leads to sustained breast milk feedings in preterm infants. Pediatr Neonatol. 2017;58:534-40.

27. Panchal H, Athalye-Jape G, Patole S. Oropharyngeal colostrum for preterm infants: a systematic review and meta-analysis. Adv Nutr. 2019;10:1152-62.

28. Garg BD, Balasubramanian H, Kabra NS, Bansal A. Effect of oropharyngeal colostrum therapy in the prevention of necrotising enterocolitis among very low birthweight neonates: a metaanalysis of randomised controlled trials. J Hum Nutr Diet. 2018;31:612-24.

29. Moher D, Liberati A, Tetzlaff J, Altman DG, Group P. Preferred reporting items for systematic reviews and meta-analyses: the PRISMA statement. BMJ (Clinical research ed). 2009;339:b2535-b.

30. Higgins JPT, Altman DG, Gotzsche PC, Juni P, Moher D, Oxman $\mathrm{AD}$, et al. The Cochrane Collaboration's tool for assessing risk of bias in randomised trials. BMJ-Br Med J. 2011;343:9.

31. Cochrane handbook for systematic reviews of interventions version 5.1.0. March 2011. https://training.cochrane.org/handbook.

32. Egger M, Davey Smith G, Schneider M, Minder C. Bias in metaanalysis detected by a simple, graphical test. BMJ (Clin Res ed). 1997;315:629-34.

33. Sharma D, Kaur A, Farahbakhsh N, Agarwal S. Role of oropharyngeal administration of colostrum in very-low-birth-weight infants for reducing necrotizing enterocolitis: a randomized controlled trial. Am J Perinatol. 2019. https://doi.org/10.1055/s-00391688817. [Published online ahead of print 14 May 2019].

34. Lee J, Kim HS, Jung YH, Choi KY, Shin SH, Kim EK, et al. Oropharyngeal colostrum administration in extremely premature infants: an RCT. Pediatrics. 2015;135:e357-66.

35. Romano-Keeler J, Azcarate-Peril MA, Weitkamp JH, Slaughter JC, McDonald WH, Meng S, et al. Oral colostrum priming shortens hospitalization without changing the immunomicrobial milieu. J Perinatol. 2017;37:36-41.

36. Sohn K, Kalanetra KM, Mills DA, Underwood MA. Buccal administration of human colostrum: impact on the oral microbiota of premature infants. J Perinatol. 2016;36:106-11.

37. Nancy A, Rodriguez P. A randomized Controlled trial of the oropharyngeal Administration of mother's Colostrum to extremely low Birth Weight Infants in the First days of life. Neonatal Intensive Care. 2011;24:31-5.

38. McCallie KR, Lee HC, Mayer O, Cohen RS, Hintz SR, Rhine WD. Improved outcomes with a standardized feeding protocol for very low birth weight infants. J Perinatol. 2011;31 Suppl 1:S61-7.

39. Burge KY, Hannah L, Eckert JV, Gunasekaran A, Chaaban H. The Protective influence of chondroitin sulfate, a component of human milk, on intestinal bacterial invasion and translocation. J Hum Lact. 2019. https://doi.org/10.1177/0890334419845338.

40. Ford SL, Lohmann P, Preidis GA, Gordon PS, O'Donnell A, Hagan J, et al. Improved feeding tolerance and growth are linked to increased gut microbial community diversity in very-low-birthweight infants fed mother's own milk compared with donor breast milk. Am J Clin Nutr. 2019;109:1088-97.

41. Wang C, Zhang M, Guo H, Yan J, Liu F, Chen J, et al. Human milk oligosaccharides protect against necrotizing enterocolitis by inhibiting intestinal damage via increasing the proliferation of crypt cells. Mol Nutr Food Res. 2019;63:e1900262-e.

42. Wang $X$, Yan $X$, Zhang L, et al. Identification and peptidomic profiling of exosomes in preterm human milk: insights into necrotizing enterocolitis prevention. Mol Nutr Food Res. 2019: e1801247. https://doi.org/10.1002/mnfr.201801247. [Published online ahead of print 8 May 2019].

43. Lönnerdal B. Bioactive proteins in human milk-potential benefits for preterm infants. Clin Perinatol. 2017;44:179-91.

44. Garofalo NA, Caplan MS. Oropharyngeal mother's milk: state of the science and influence on necrotizing enterocolitis. Clin Perinatol. 2019;46:77-88.

45. Silvestri L, van Saene JJ, van Saene HK, Weir I. Topical chlorhexidine and ventilator-associated pneumonia. Crit Care Med. 2007;35:2468

46. Vollman KM. Ventilator-associated pneumonia and pressure ulcer prevention as targets for quality improvement in the ICU. Crit Care Nurs Clin North Am. 2006;18:453-67.

47. Picaud JC, Buffin R, Gremmo-Feger G, Rigo J, Putet G, Casper C. Review concludes that specific recommendations are needed to harmonise the provision of fresh mother's milk to their preterm infants. Acta Paediatr. 2018;107:1145-55.

48. Landers S, Updegrove K. Bacteriological screening of donor human milk before and after Holder pasteurization. Breastfeed Med. 2010;5:117-21.

49. Schanler RJ, Fraley JK, Lau C, Hurst NM, Horvath L, Rossmann SN. Breastmilk cultures and infection in extremely premature infants. J Perinatol. 2011;31:335-8.

50. Zhu XL, Tang XG, Qu F, Zheng Y, Zhang WH, Diao YQ. Bifidobacterium may benefit the prevention of necrotizing enterocolitis in preterm infants: a systematic review and meta-analysis. Int J Surg. 2019;61:17-25.

51. Rodriguez NA, Vento M, Claud EC, Wang CE, Caplan MS Oropharyngeal administration of mother's colostrum, health outcomes of premature infants: study protocol for a randomized controlled trial. Trials. 2015;16:453.

52. Sadeghirad B, Morgan RL, Zeraatkar D, Zea AM, Couban R, Johnston BC, et al. Human and bovine colostrum for prevention of necrotizing enterocolitis: a meta-analysis. Pediatrics. 2018;142: e20180767. 